Linguistique, littérature, didactique

\title{
Vivre poème en classe : Apollinaire et la dinette
} rose

Living the poem in class: Apollinaire and the pink dinette

\section{Frédérique Cosnier-Laffage}

\section{(2) OpenEdition}

\section{Journals}

\section{Édition électronique}

URL : http://journals.openedition.org/pratiques/5207

DOI : $10.4000 /$ pratiques.5207

ISSN : 2425-2042

Éditeur

Centre de recherche sur les médiations (CREM)

Référence électronique

Frédérique Cosnier-Laffage, «Vivre poème en classe : Apollinaire et la dinette rose », Pratiques [En ligne], 179-180 | 2018, mis en ligne le 31 décembre 2018, consulté le 19 avril 2019. URL : http:// journals.openedition.org/pratiques/5207; DOI : 10.4000/pratiques.5207

Ce document a été généré automatiquement le 19 avril 2019

(c) Tous droits réservés 


\section{Vivre poème en classe : Apollinaire et la dinette rose}

Living the poem in class: Apollinaire and the pink dinette

Frédérique Cosnier-Laffage

\section{Du sujet à la transsubjectivation par le poème}

\section{Déplorations}

1 La déploration de l'approche formaliste et techniciste des textes littéraires en classe de français dans le secondaire apparait comme un fait avéré (Langlade, 2004), relevant quasiment du poncif (Rouxel, 2007). Les effets regrettables proviennent d'une relégation de l'apprenti sujet lecteur et scripteur à la porte des œuvres. Même les derniers programmes de cycle 4 semblent tenir compte d'un manque de motivation des élèves, pris entre "résignation désabusée, dérision, indifférence, refus » (ibid., p. 67). La lecture analytique se trouve ainsi reléguée, comme subrepticement, dans le descriptif des trois modes de lecture indiqués pour la progression pédagogique: «lecture intégrale", «lecture cursive», « lecture analytique» (MÉNESR, 2015, p. 236). Les « attendus de fin de cycle» incluant l'activité d'interprétation n'osent plus s'appuyer que sur «quelques outils d'analyse simples » (ibid., p. 234). On pourrait considérer les activités préconisées de «reformulations, verbalisation des représentations mentales", la «formulation de jugements de goût » et même l'apparition des « mises en voix et théâtralisation » (ibid., p. 234-235) comme faisant écho aux théories de la réception et du sujet lecteur, à qui on cherche à faire une place. Les orientations de toutes les activités du dire-lire-écrire prendraient donc en compte ce déni d'expérience (Canvat, 2007, p. 27) que constituent les dérives de la lecture savante. Cependant, la préconisation d'une lecture dite littéraire, qui était supposée engager le lecteur, n'est pas exempte des mêmes risques de distanciation produits par une démarche toujours « rationalisante » (ibid., p. 28) ${ }^{1}$.

2 Il semble donc urgent de s'interroger sur les fondements d'un problème qui finit par prendre la forme d'une véritable aporie dès lors que l'on se met en quête du sujet dans 
l'approche du texte littéraire, a fortiori dans le cadre scolaire. Comment le prendre en compte? Et de quel sujet s'agit-il véritablement? On peut se demander en quoi la didactique de la poésie pourrait être un levier puissant pour renouveler les conceptions du sujet, lui qui semble "résister à toute théorisation et, peut-être, même, à toute véritable domestication » (Langlade \& Rouxel, 2004, p. 12). Comme l'annonce M. Favriaud dans la préface de notre livraison, il s'agit bien de questionner des théories qui se sont constituées en oubliant le lien pourtant irréductible qui existe entre constitution du sujet et langage, ce que révèle au plus haut point la poésie (Laplantine, 2011).

Le concept permettant de sortir de l'impasse pourrait être celui de sujet du poème, investi par G. Dessons, S. Martin et M. Favriaud, dans la lignée d'H. Meschonnic (1982a ; 1995), pour ne plus confondre subjectivisme et subjectivation (Martin, $2013 ; 2014$ ), cette dernière demandant « de penser la relation du point de vue d'une anthropologie du langage où du sujet s'invente dans et par la relation (je-tu), c'est-à-dire l'historicité continuée d'une transsubjectivation, l'oralité infinie d'une écriture-lecture", selon S. Martin ${ }^{2}$. L'accent est mis sur un processus plus que sur son résultat: l'activité de transformation du sujet, dans et par le langage spécifique de l'œuvre.

\section{Les problèmes de l'interprétation}

4 La persistance du paradigme de la lecture comme négociation du sens par l'interprétation institue, tout en ménageant des interactions, deux pôles séparés, texte et lecteur, voire trois, avec la communauté interprétative. L'attention aux phénomènes de l'intersubjectivité (à partir d'É. Benveniste et $\mathrm{H}$. Meschonnic) et du dialogisme bakhtinien (voir par exemple Boré, 2011) semble attestée, cependant le sujet demeure pris dans la tenaille d'une "tension" entre deux entités. Même considérés comme ouverts et dynamiques (dans le concept de « texte du lecteur » où le texte est inachevé, construit par le lecteur autant qu'il est construit par lui, Rouxel, 2007, p. 69-70), les deux pôles relèvent toujours de «droits» distincts. Si bien qu'A. Rouxel se demande (ibid., p. 71) : «jusqu’à quel point le sujet lecteur peut-il métamorphoser le texte ? Quelles limites donner à cette reconfiguration? " et précise que ces questions «s'imposent particulièrement dans le cadre de la classe et de l'évaluation des conduites interprétatives ", car elles "évoquent en creux l'existence d'une norme qui invite au respect des "droits du texte" et rappellent la nécessité de développer chez les élèves réflexivité et recul critique ». Où l'on retrouve la nécessité d'une distance, alors même que l'on tentait de rétablir une proximité...

Cette séparation persistante s'accompagne d'une conception des "débordements de subjectivité », qui va de «l'erreur de lecture » au « délire » en passant par les « caprices » combattus par F. Brunetière et G. Lanson et rappelés par A.Compagnon (cité par Langlade, 2004). Certes, ces marques d'expression sont désormais prises positivement, dans une "didactique de l'implication", comme marques d'implication toujours préférables à une "posture d'extériorité» (Rouxel 2007, p.72), ou marques des "capacités de l'enfant» à entrer dans une "interaction entre deux sujets, auteur et lecteur » (Favriaud, Vinsonneau \& Poletto, 2017, p. 85). Elles déterminent dès lors un des gestes professionnels de l'enseignant qui doit aménager un espace de liberté accueillant et bienveillant au sein de la communauté des apprentis lecteurs, dans la classe considérée comme "lieu d'émergence et de confrontation de lectures subjectives» (Rouxel, 2007, p. 72). L'écopoésie, avec la mise place d'une série d'aménagements et d'outillages dans l'espace géographique de la classe, ainsi que de gestes propices, kaïros et bonification 
(Favriaud, Vinsonneau \& Poletto, 2017, p. 90 et 126; Vinsonneau, 2017, p. 184-221; Favriaud et al., 2009), s'inscrit dans cette perspective.

6 Cependant, on peut aussi se demander s'il ne faudrait pas envisager une autre voie que celle du sujet lecteur, scripteur, didactique, scolaire, au profit du concept de sujet du poème. Celui-ci se révèle comme un processus dynamique de subjectivation voire de transsubjectivation, où ce ne sont plus tant les deux pôles du texte et de sa réception qui importent mais ce qui se passe précisément entre les deux, dans l'activité même du langage en poésie.

\section{Au-delà des deux pôles : la transsubjectivation par l'oralité}

7 La poétique d'H. Meschonnic remet fondamentalement en question les théories du sujet. Nous présenterons tout d'abord la constellation conceptuelle qui relie sujet du poème, subjectivation et transsubjectivation.

- Dans «sujet du poème", le mot "poème", ne désigne pas le genre littéraire mais est « invention d'une forme de vie par une forme de langage et invention d'une forme de langage par une forme de vie» (Meschonnic, 2005, p. 257). Le sujet du poème «n'est pas l'individu. L'auteur. C'est l'activité même de subjectivation d'un discours, d'une pratique. [...] Ainsi le sujet du poème se diversifie infiniment.» (Meschonnic, 2001, p. 43). Le sujet du poème n'étant pas un produit, c'est une activité transformante.

- Ce sujet est même plutôt cette subjectivation même : «Il y a une poétique du rythme quand l'organisation du mouvement de la parole dans l'écriture est le fait d'un sujet spécifique, qu'on appellera le sujet du poème. Ce sujet fait que l'organisation du langage est une subjectivation générale, et maximale, du discours, telle que le discours est transformé par le sujet et que le sujet advient seulement par cette transformation même » (Dessons \& Meschonnic, 1998, p. 43). La subjectivation ne se fait pas ailleurs que dans et par l'œuvre, qui transforme le langage en même temps que le sujet, sans quoi elle ne serait pas « œuvre ».

- S'il y a subjectivation et transformation réciproque, du sujet par l'œuvre et de l'œuvre par le sujet, le concept va cependant au-delà de l'intersubjectivité. Il n'y a pas deux sujets séparés, mais le sujet du poème est cette sorte de troisième zone, processus formé par la chaine des réénonciations du poème: "Si une écriture produit une reprise peut-être indéfinie de la lecture,_sa subjectivité est une intersubjectivité, une trans-subjectivité. [...] Cette écriture est une énonciation qui n'aboutit pas seulement à un énoncé, mais à une chaîne de ré-énonciations. C'est une énonciation trans-historique, trans-idéologique » (Meschonnic, 1982a, p. 86-87). G. Dessons (2004, p. 270) précise que c'est « le sens d'une conception de la littérature comme passeuse de manières, fondée sur l'éthique non intersubjective, mais transsubjective de l'artlangage $»$.

8 Comme souvent chez H. Meschonnic, la conceptualisation est intégrative et spiralaire. C'est ainsi que la «subjectivation générale, et maximale, du discours » dont parlent G. Dessons et H. Meschonnic, que l'on retrouve comme «subjectivisation » chez H. Meschonnic seul, est aussi associée à la notion de rythme, elle-même associée à celle d'oralité : « Le rythme, comme sémantique, et oralité, est une subjectivisation spécifique du langage » (Meschonnic, 1982a, p. 660). L'oralité est "notation du "mouvement de la parole dans l'écriture" " (Meschonnic 1982b, p. 18), et elle "échappe à la simple opposition avec l'écrit» (Meschonnic, 1982a, p. 706), car la "pluralité des modes de signifier, et des inscriptions de l'énonciation, dissémine l'oralité dans l'écrit comme dans le parlé » (Meschonnic, 1982b, p. 16). 
Ce rappel épistémologique entraine une orientation forte en didactique. Il faudrait chercher plutôt la signifiance que le sens, à travers des activités tant orales qu'écrites, à la recherche d'un rythme :

Je définis le rythme dans le langage comme l'organisation des marques par lesquelles les signifiants, linguistiques et extra-linguistiques (dans le cas de la communication orale surtout) produisent une sémantique spécifique, distincte du sens lexical, et que j'appelle la signifiance : c'est-à-dire les valeurs propres à un discours et à un seul. Ces marques peuvent se situer à tous les «niveaux» du langage: accentuelles, prosodiques, lexicales, syntaxiques. (Meschonnic, 1982a, p. 216-217).

Dès lors, le paradigme interprétatif traditionnel peut être remis en question, car «le sujet, le sens sont flottants dans le rythme » (ibid., p. 93). L'hypothèse est que l'on peut expérimenter des activités qui favoriseraient l'activité avec les textes, en tenant compte de ces sujets et sens « flottants ».

11 C'est dans cette perspective que les activités dites de réénonciation peuvent être envisagées. Nous nous appuierons sur l'incitation de S. Martin (2014, p. 107-114) à « Faire œuvre avec les œuvres » : il s'agit «non d'expliquer les textes mais de les reformuler, de les approprier dans sa voix, ses gestes, ses écrits individuellement et collectivement », de favoriser «Implication personnelle et dynamique collective » dans le but de «favoriser les parcours individuels autant que la construction d'une culture commune (ibid., p. 107).

12 Je vais maintenant présenter une partie d'une expérience menée en classe de $4^{\mathrm{e}}$, autour des Calligrammes. Poèmes de la paix et de la guerre (1913-1916), de G. Apollinaire, en me concentrant sur cette triple problématique: que peut-on attendre des activités de réénonciation qui prendraient la place des activités interprétatives traditionnelles? Le concept poétique de transsubjectivation est-il repérable en didactique dans les différentes facettes du dire des élèves, et comment (selon quels indicateurs)? Quels en sont les apports didactiques? Chaque partie portera sur la recherche de différents types de repérages d'une transformation en acte de l'élève par le poème.

\section{Chercher l'oralité des poèmes par le lien du dire-lire- écrire}

Le recueil Calligrammes. Poèmes de la paix et de la guerre (1913-1916)3 , a été choisi car il inscrit le poème au cœur de la vie : comme il le déclare dans son texte manifeste de 1918, l'esprit nouveau que recherche G. Apollinaire est celui qui pourrait « explorer tous les domaines propres à fournir une matière littéraire qui permette d'exalter la vie sous quelque forme qu'elle se présente». Exaltation de la vie dans la guerre, de ses surprises, de ses découvertes, de l'expérimentation, ce principe fait écho à celui que nous nous posons au plan didactique. C'est également un recueil qui fait lien, comme l'indique le premier titre du recueil, entre tradition et modernité, entre les hommes, entre les discours (poèmesconversations), entre les différentes postures énonciatives (poèmes simultanés créant polyphonie), entre les arts (les calligrammes et plus globalement la recherche sur « les artifices typographiques" et le "lyrisme visuel», l'aventure du vers libre, le tout participant de la recherche d'une véritable "synthèse des arts, de la musique, de la peinture et de la littérature ». Cette exaltation de la vie dans et par une poétique en actes me semblait propice à explorer comment "Écrire un poème, c'est faire la vie », et 
comment «Lire un poème, c'est sentir la vie qui nous traverse et être transformé par lui » (Meschonnic, 2006, p. 12).

La séquence a consisté en cinq séances (une quinzaine d'heures de cours), organisées autour des activités lancées par S. Martin, auxquelles j'ai rajouté une séance d'introduction :

- Une séance de «mise en route » autour des représentations et souvenirs des élèves avec les poèmes (que je ne présenterai pas ici, au profit des activités réalisées avec les textes euxmêmes, mais qui se justifiait en termes de gestes professionnels: "instauration d'un climat ", " mise en scène des savoirs » en partant "de ce que l'élève sait faire pour le conduire à progresser sur un autre plan » selon A. Jorro et H. Crocé-Spinelli (2010, p. 128 et 130). En termes de planification pédagogique, mon but était aussi de comparer les contenus de cette étape d'amorce avec les propos réflexifs de fin de séquence, dont la trace était attendue dans les conclusions des carnets. Comparaison importante pour repérer d'éventuelles marques de transsubjectivation).

- Deux séances avec activités d'échelles ${ }^{4}$.

- Une séance de mise en voix.

- Une séance d'écriture créative.

Les activités de recherche documentaire ont été réalisées à la maison. L'ensemble de ces activités fournit la matière pour la réalisation d'un carnet de lecture, dont le contenu et les attentes ont été présentés en première séance. Il a été expliqué aux élèves que cette démarche avait pour but de sortir des traditionnelles explications de texte, de ne pas se référer à des questionnaires de lecture, mais de développer chez eux une expérience singulière, originale, dont ils auraient à inventer eux-mêmes les contenus selon des choix et des questionnements personnels.

16 Je présenterai dans la prochaine étape les enjeux du carnet de lecture, puis je m'attacherai aux activités d'échelle et de mise en voix, en tentant de trouver à chaque fois dans les données recueillies (enregistrements sonores et vidéo, productions écrites) des indicateurs aptes à nourrir la problématique annoncée. Les activités de la séance d'écriture créative proprement dite ne seront pas traitées ici, faute de place, mais on verra que les échelles intègrent déjà une telle forme d'écriture, avec le texte, le concept d'oralité incitant toujours à lier oral et écrit. Le carnet de lecture est quant à lui une forme d'écriture mixte, contenant les textes créatifs, et des textes réflexifs.

\section{Voix des lectures : le carnet comme relation des expériences}

17 L'hypothèse était que le carnet de lecture, s'il permettait d'écrire les traces de toutes les activités, orales comme écrites (et pas seulement comme recueil d'impressions au fil de la lecture, comme le préconisent M. Favriaud, M. Vinsonneau \& M. Poletto, 2017, p. 88-89), serait un dispositif à même de créer un dire généralisé du lire-écrire, ce que $\mathrm{S}$. Martin (2018) nomme un "essai de voix». J'ai expliqué aux élèves qu'il devrait contenir la présentation d'un choix d'activités réalisées, accompagné de réflexions, questionnements, recherches personnelles sur l'auteur, le lexique, le calligramme, et bien sûr, d'écrits créatifs. De ce point de vue, le carnet de lecture peut ne pas être qualifié comme écrit « intermédiaire » (Chabanne \& Bucheton, 2002), mais bien comme un écrit de transsubjectivation. Je rejoins par là l'idée d'une « poétique du lire-écrire, qui est un faire esthétique et éthique, physique et réflexif " (Favriaud, Vinsonneau \& Poletto, 2017, p. 142). Avec ce livre des lectures, les élèves, comme les poèmes développant leur oralité, 
ont un lieu pour développer la leur. En quoi s'explique la formule de S. Martin, «faire œuvre avec les œuvres ».

\section{Ouvrir les lectures}

\section{Travail collectif oral}

Je propose, lors de la deuxième séance (comme déjà dit, la première était consacrée à un rappel des souvenirs des élèves avec les poèmes), un travail sur le titre général. Sur la couverture de l'édition scolaire, on lit "Calligrammes ", et en page intérieure le titre complet, « Poèmes de la paix et de la guerre - 1913-1916 ». Les élèves sont invités à réagir sur ce titre. Il s'agit de motiver des questionnements qui proviennent des lecteurs et de leur relation au texte, non de grilles de lecture qui orienteraient l'entrée dans le texte, au risque de parasiter l'engagement. Cela peut aller du mot " calligrammes » à l'évocation de thématiques (paix et guerre), à leur ordre d'apparition (paix et guerre vs guerre et paix), aux dates (1913-1916 n'est pas 1914-1918). Quelques échanges enregistrés (avec P pour professeur) :

(P) Quel est le titre marqué sur la couverture?

$>$ Calligrammes

$>$ Ça fait penser à calligraphie

$>$ Ça veut dire quoi en latin?

(P) Pourquoi tu penses au latin?

$>$ Parce qu'on découpe

$>$ Moi je sais, c'est une écriture

$>$ Avec une plume, de l'encre

> Ça s'écrit avec le calame. Un bout de bambou

[observation d'images de calligraphies en différentes langues par vidéoprojecteur]

> on peut écrire dans tous les sens

[phase d'explication grâce à l'encart documentaire que les élèves trouvent p. 27

Calligramme : néologisme croisant idéogramme et calligraphie]

(P) Mais quel est le titre entier ? Feuilletez un peu le livre...

> « Poèmes de la paix et de la guerre, 1913-1916 "

(P) Est-ce la même chose?

> Calligramme c'est de la grammaire, Poèmes de la paix et de la guerre c'est plus poétique.

> C'est peut-être des poèmes écrits pendant la guerre

$>$ C'est des sujets qui ont été mélangés : la paix et la guerre

$>$ C'est opposé

(P) Oui, mais il y a le « et »

$>$ Pour la paix?

> Aussi sur la couverture il y a un soldat... Peut-être qu'il se repose... Peut-être qu'il

écrit

$>$ Si ça se trouve il a repris des poèmes que les soldats écrivaient

$>$ On voit « oh mes amis » ceux qui sont restés là-bas ou qui n'étaient pas là-bas

$>$ p. 84-85 on a des images de la guerre

$>$ p. 32 on voit un oiseau

$>$ p. 7 y'a une photo de GA blessé à la tête

$>$ Ah je sais! Il était soldat et il a écrit des poèmes pendant la guerre

$>$ Il a été blessé ? Il est pas mort?

Cette première phase d'échanges s'apparente à un début de débat interprétatif, à partir du lexique (un mot inventé par G. Apollinaire), et le déborde immédiatement, dans une forme des plus dynamiques. Cependant je constate qu'il ne fait pas suite à une activité de 
type questionnaire mais à la présentation en classe d'une modalité de travail beaucoup plus ouverte (le carnet de lecture). J'accueille ces propositions comme autant de marques de motivation immédiate, et également parce que certaines d'entre elles vont permettent de tracer un sillon dans la signifiance, notamment autour de la question du mélange des registres et aussi de cette étonnante remarque «c'est de la grammaire »/«c'est plus poétique », qui questionne d'emblée la signifiance. L'aspect formel indiqué par "Calligrammes » semble relié à la grammaire, sans doute aussi par le "gramm » qu'ils ont en commun. L'indication thématique (paix et guerre) infère une indication de genre (poétique). Le tout dessine deux ensembles distincts (grammaire/genre-thème, comme forme/fond), mais que l'élève voit reliés dans le titre. Par ailleurs, je ramène les élèves vers l'observation du texte, non pour faire valoir ses «droits ", mais parce que l'activité d'écriture des échelles doit faire travailler le sujet du poème, comme rapport entre lecture et écriture.

J'invite à lire les titres du sommaire, à observer disposition et typographie, et à voir comment l'ensemble est organisé :

[Lecture par deux élèves]

(1) On dirait qu'il y a plusieurs chapitres, des grands titres avec plusieurs poèmes dedans

(P) Oui, des grands titres avec plusieurs poèmes dedans, tu appelles ça chapitres, est-ce que tu peux donner des exemples?

(1) Il y a « Obus couleur de lune », avec quatre poèmes

(P) Oui, est-ce vous pouvez me donner d'autres exemples de ces chapitres, qu'on peut appeler aussi des sections, en poésie?

(2) il y a "Ondes », «Etendards », "Cases d'Armons », «Lueurs des tirs », «Obus couleur de lune » et « La tête étoilée »...

(P) Oui donc on a 6 sections et plusieurs poèmes dans chaque (écriture au tableau). Alors étant donné tout ce qu'on a dit hier [sur le titre principal], je pense que vous pouvez réagir à ces titres (je les relis) : à quoi ils peuvent vous faire penser ?

(2) Ça fait penser un peu à ce qu'on avait vu avec Victor Hugo et ses titres, il y a l'ombre et la lumière

(P) Ah d'accord, toi ça te fait penser à VH, oui pourquoi ?

(2) Parce que là on peut voir qu'il y a une part d'ombre et de lumière : les lueurs des tirs, les obus couleur de lune...

(P) Ah oui lueur... lune

(3) Mais c'est normal madame, vu qu'ils font la guerre, c'est à cause de la fumée et tout... ça fait qu'il y aurait besoin de la lumière peut-être... il y a trop de trucs on voit rien, vu qu'on court il y a de la poussière, on parle de fumée...

(P) Oui ça peut être la fumée des combats, des tirs, de la vision brouillée par tout ça

(4) Pourquoi Case d'Armons Madame?

(P) Ah... Pourquoi... On cherchera... Notez ça...

Les hypothèses interprétatives se teintent de remarques qui pourraient constituer des indices de transsubjectivation, à travers trois ensembles de questionnement :

- Mobilisation des savoirs (sur la narratologie, les genres, les œuvres patrimoniales), chez (2) qui repère un rythme, dans sa composante lexicale, avec associations apparemment contradictoires (ombre et lumière). Cependant, il n'y a pas de sensibilité apparente à la dimension sonore (paronomase lueur - lune, qui n'est pas la même chose que ombre et lumière).

- Déclenchement de l'imaginaire par une mise en relation avec la "protonarrativité » des poèmes (Favriaud, Vinsonneau \& Poletto, 2017, p.74): peut-être un prolongement du questionnement générique amorcé durant la première séance, où certains élèves avaient rappelé que les poèmes peuvent raconter des histoires. La transsubjectivation peut-elle être 
comme un bougé des représentations, ici sur les genres, où (3) permet déjà d'éviter un des clichés opposant poésie à récit, voire poésie à roman (ibid., p. 56) ?

- Stimulation de la curiosité lexicale (et/ou phonétique ?) : avec le « Case d'Armons » de (4).

\section{Travail individuel d'écriture : les échelles}

Je propose ensuite de passer au travail individuel écrit. Selon le principe des «échelles » proposé par S. Martin (2014, p. 112), je demande de procéder à des regroupements libres parmi les différents titres et de trouver de nouveaux titres à ces ensembles.

Nous obtenons :

- «violence » (avec « De la batterie de tirs", "Guerre », "Lueurs des tirs ", «Les feux du bivouac », «Fumées », «La Colombe poignardée et le jet d'eau »), qui correspond à d'autres séries repérées sous le titre de «guerre » ou « les combats ». Un élève précise «j’ai mis les collines dedans parce que j'ai regardé et en fait ça parle de guerre », un autre dit «il y a aussi l'adieu du cavalier », deux autres dialoguent: " on peut mettre "Du coton dans les oreilles" parce que les tirs ça fait du bruit »

- "paysage et lieux» : avec «Il pleut », «Les collines ", " Dans l'abri caverne », "Lundi rue Christine », complété par un autre élève, «Paysage » et par «L'adieu du cavalier», qui « parle de lieux de la guerre»

- «musique/chant», «de la batterie de tirs» (avec contre-sens sur «batterie», que je signale), «Le chant d'amour » et « Fêtes »

- "le positif» $[$ sic $]$ avec «La fête ", "Le chant d'amour", «Eventail des saveurs", «Souvenir », « La jolie rousse »

- «La retombée » [sic], avec « Il pleut », « Fusée », « Fumée »

On repère des balayages par motifs thématiques assez traditionnels au début, avec recours à une sorte de relevé de champ lexical (violence, guerre, combats, paysages), donnant lieu à la formulation d'hypothèses dans une recherche de cohérence (le sens ?). Cependant, ne peut-on voir les embryons d'une écoute du rythme, c'est-à-dire là encore, de signifiance, lorsque les élèves s'orientent, dès la troisième et la quatrième liste sur la musique et «le positif», vers ce que la guerre devient dans le langage apollinarien, comme recherche d'un lyrisme total, mobilisant le chant et tous les sens, le corps souffrant mais aussi exalté, l'amour, le temps présent et celui du souvenir? Plus encore, c'est le regroupement effectué dans la cinquième liste, associé à cette invention d'écriture, «la retombée ", qui pourrait être perçu comme début, même très modeste, de transsubjectivation: une lecture-écoute et observation du texte dans son mouvement, déclenchant dans le langage un imaginaire plastique et lexical. Ces premiers résultats peuvent paraitre infimes, eu égard au concept convoqué, mais il ne faut pas oublier qu'ils ne constituent qu'une partie des résultats obtenus au terme de la séquence entière. Ils sont confirmés par exemple dans les poèmes écrits par les élèves, que je n'analyse pas ici, me concentrant sur des activités peut-être moins répandues et surtout sur le principe global de mise en œuvre. 


\section{Mettre en voix et en corps}

\section{Vivre l'oralité du poème} corps audible dans le texte n'est pas seulement voix, rythme, ton, oralité. Il est tout cela à la fois et en même temps moins que cela. Il est désir de corps et désir de voix incarnée, corps désigné et corps introuvable » (cité par Martin, 2017, p. 75). Insistant sur cette voix du texte qui s'apparente plus à une recherche de voix, il met en avant l'idée d'un désir de corps. S. Martin (2014, p. 112) voit quant à lui les «essais de théâtralisation " comme autant d'occasions pour « jouer l'œuvre pour de bon », afin de " visualiser, d'entendre, de [faire] résonner l'œuvre ». Cette piste semble particulièrement intéressante, d'autant qu'une des élèves a suggéré que les ondes étaient «quelque chose qu'on ne voit pas ». Le travail du corps est primordial pour entendre l'oralité, car elle est un mouvement traversant de l'écrit vers oral et inversement, du corps de l'auteur au corps d'écriture, et vers le corps du lecteur. D'ailleurs, je repère que les élèves parlent d'ondes de choc, d'électronique, de vibrations, et que cela suscite agitation physique et rires, comme autant d'indices d'implication immédiate dans l'activité. Un élève se demande également «comment on va faire quand c'est un peu comme des dessins »... Dans les premières réactions après ma lecture des poèmes, les élèves réagissent :

> dans « La cravate et la montre », c'est le dessin d'une cravate et... (la forme de la montre n'est pas immédiatement repérée, il faut passer par l'explication de la montre à gousset)

$>$ On a du mal à lire

$>$ Il y a des choses qui veulent rien dire

> dans « Lettre-Océan » il y a des petites lignes comme ça, peut-être ça représente la mer, et il y a un soleil en-dessous de la mer mais c'est pas logique !

$>$ Il y a aussi une cigarette allumée qui fume dans « Paysage » et un bonhomme

Je demande s'ils auraient lu comme moi le calligramme « Paysage » [que j'ai lu dans cet ordre: «voici la maison où naissent les étoiles et les divinités »]. Une élève dit non, proposant «v /oi /la » plutôt que « v /oi/Ci », avant de se reprendre immédiatement, à cause de l'impossible utilisation du «la», qui ne porte pas d'accent. Ensuite, dans un 
brouhaha difficilement transcriptible, s'essaient des lectures du « bonhomme » : « amants couchés ensemble, vous vous séparerez mes membres" / " couchés ensemble, amants, vous vous séparerez » / " vous vous séparerez mes membres ».... Le « vous » désigne les amants ou mes membres, ou les deux, ce que les élèves ne décident pas.

Conclusion des élèves : «il y a plusieurs interprétations possibles", "ça nous force à réfléchir ", "mais Apollinaire il a vraiment écrit comme ça?», "ça fait penser aux numéros qu'on relie quand on est petit et ça donne une image", "comme on doit chercher comment lire, ça nous donne un rythme de lecture (parce qu'on cherche) », « tu peux lire tout et après tu mets dans un autre sens ", « il y a du mouvement ».

Je lis ici l'activation de questionnements syntaxiques (voici/voilà), de modalités de lectures, qui reviennent à se demander : qu'est-ce que lire? Toutes questions que les calligrammes posent. Non seulement "poèmes-dessins", proposant «un rapport univoque entre un référent et un signe visuel, mais relevant «d'une construction multiple, variée, débordant largement les catégories de l'idéogramme ", les calligrammes de G. Apollinaire font que tout « le protocole traditionnel de la lecture est bouleversé » (Debon, 2004, p. 14-15) car la figuration des différents éléments « oblige à les considérer simultanément » (Butor, 1966, p. 17). Au-delà d'une dimension mimétique, ils sont « une recherche de la mise en espace du texte " (Dessons, 2011, p. 73) et finissent par être indissociables de leur objet, véritables formes-sens, mots "moulés, fondus dans la chose " (Bassy et al., 1980, p. 55). Parmi les poèmes de la section « Ondes ", dont tous ne sont pas des calligrammes, ce sont pourtant bien ces derniers que les élèves ont majoritairement choisis de mettre en voix, comme si leur corps devait participer entièrement de cette écoute d'un nouveau corps-langage, et allait leur donner les réponses aux questions de syntaxe évoquées plus haut.

\section{Quand lire fait « vivre poème »}

Parmi les essais présentés, deux mises en voix de «La cravate et la montre sont proposées » (voir figures 1 à 3 ).

1. Recherche sur la contextualisation (disposition, accessoires)

\begin{tabular}{|l|l|}
\hline Groupe 1 & Groupe 2 \\
\hline $\begin{array}{l}\text { Deux tables, un élève lit le journal, l'autre rêve, le } \\
\text { troisième passe de l'un à l'autre }\end{array}$ & $\begin{array}{l}\text { Trois personnages en train d'écrire au } \\
\text { café autour d'une table }\end{array}$ \\
\hline $\begin{array}{l}\text { C tient un journal troué à la place des yeux, T regarde } \\
\text { C à travers en disant l'ensemble } 3\end{array}$ & Montre à gousset \\
\hline
\end{tabular}

\section{Aspects linguistiques}

- Sur la cohérence textuelle. Le groupe 1 propose une lecture assez éclatée, faisant alterner des blocs pris isolément et se répondant : le texte est compris de manière très polyphonique, comme si deux voix s'envoyaient l'une à l'autre des pensées ou des souvenirs sans relation immédiate, les blocs 1 à 10 s'enchainant par mouvement descendant, les suivants semblant perdre leur organisation circulaire, renforçant l'éclatement. Le groupe 2 lit par grands ensembles et avec moindre alternance des voix : lecture moins polyphonique, comme la 
vision d'une seule instance narratrice démultipliée en trois voix, s'unissant à la fin. Chaque bloc est perçu comme ayant une logique interne.

- Sur le registre, suggéré par l'intonation: plutôt comique (coq à l'âne) pour le groupe 1, pathétique pour le groupe 2, surtout lorsque $\mathrm{V}$ lit son dernier ensemble en détachant les mots. La nature syntaxique des constituants semble avoir provoqué le regroupement des éléments: tous des noms communs ou propres, alors que les constituants des autres ensembles sont au minimum des GN, au maximum des propositions. Plus courts, les constituants du dernier ensemble semblent donc avoir été lus dans une progression dramatique, avec une sorte d'effet de chute, que suggère une intonation déplorative. « Agla » est lu dans un murmure (et c'est très beau...)

3. Aspects motivationnels et émotionnels

- Participation, valorisation et plaisir. Deux élèves, très en difficulté, choisissent le calligramme « Du coton dans les oreilles», qui leur parait plus facile car comportant peu de mots (critère discutable) et la réalisation, simple, même sans réelle implication marquée, est une première entrée dans la recherche du rythme : répartition des blocs, prosodie. Certains disent leurs difficultés, mais toujours dans des formulations où le rire et la recherche sont associés: «nous avons bien rigolé mais nous avions du mal à mettre en mouvement les paroles. / j'ai travaillé dessus et on ne savait pas dans quelle ordre il fallait lire » [sic], écrit Théo dans son carnet de lecture, ce qui confirme les pistes développées ci-dessus.

Figure 1. Le poème "La Cravate et la montre »

\section{La Cravate et la montre}

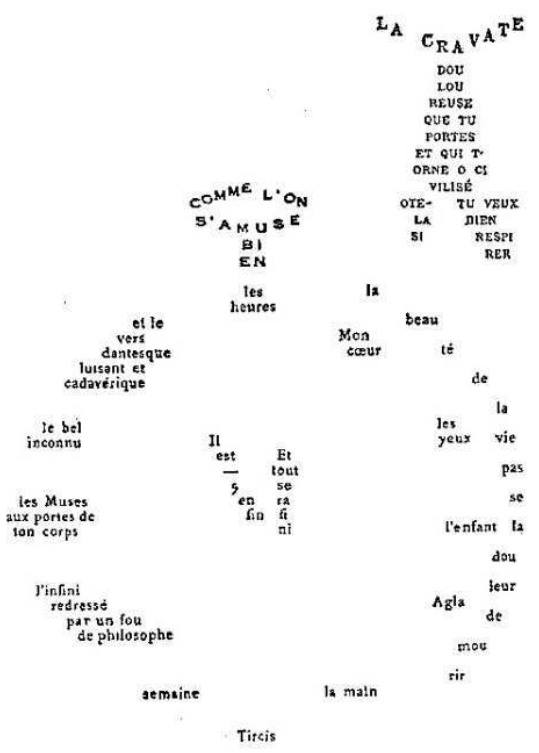


Figure 2. Mise en voix de " La Cravate et la montre », Groupe 1

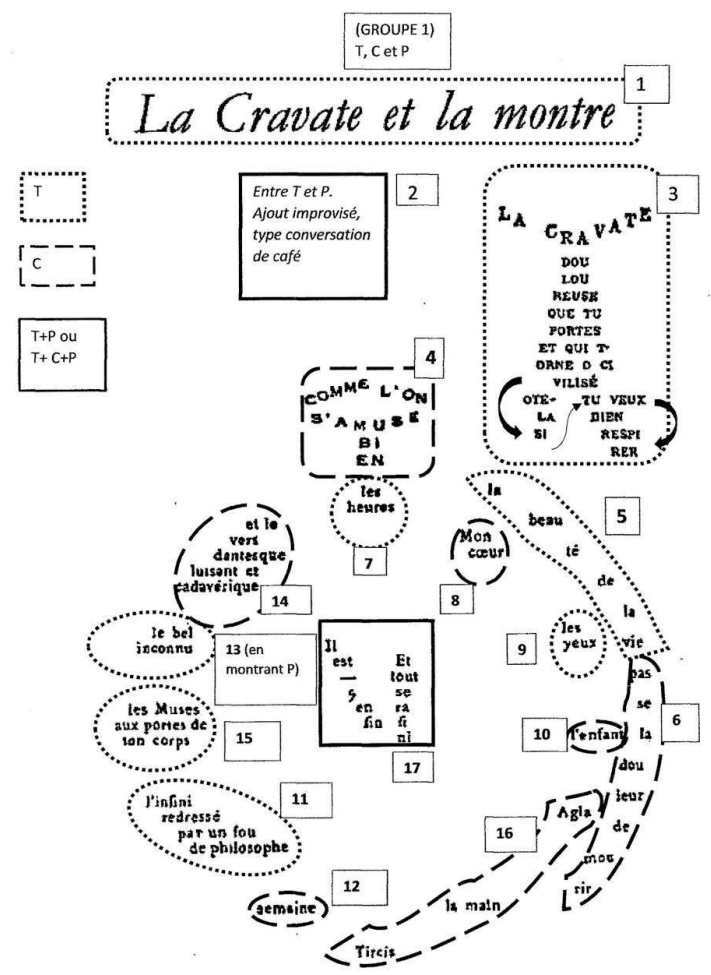

Figure 3. Mise en voix de « La Cravate et la montre », Groupe 2

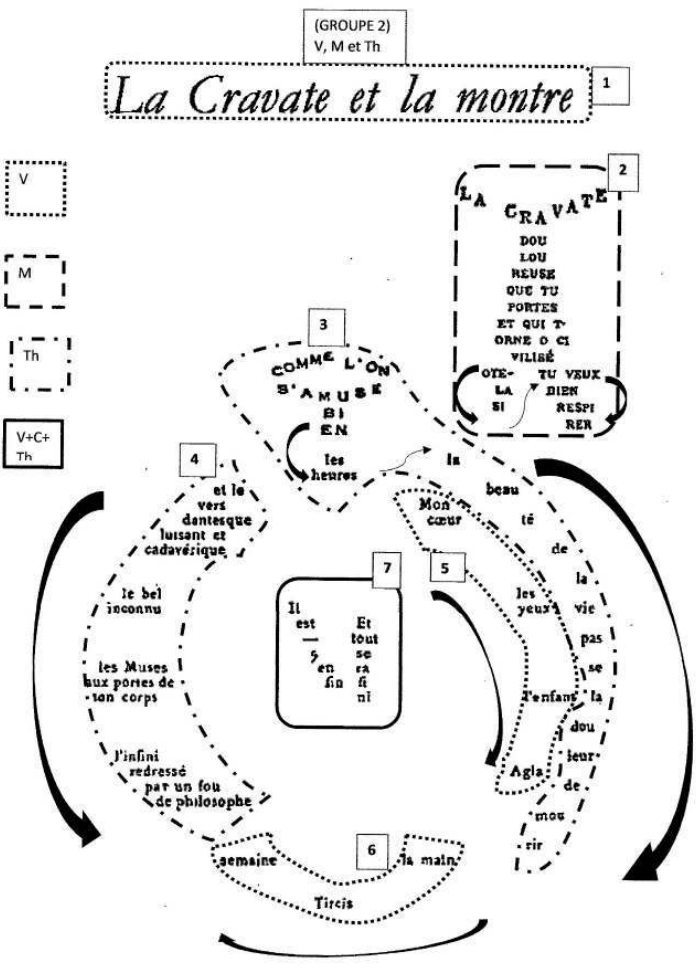




\section{Conclusion}

On pourrait regretter que les analyses présentées ici n'aient pas été confrontées à des explications récoltées auprès des élèves sur leurs choix respectifs. Mais on peut considérer que la mise en œuvre du carnet de lecture appelait de tels commentaires. Il est vrai que ces derniers se sont révélés encore en majorité assez descriptifs, et peu réflexifs. Cependant, de nombreuses bribes réflexives sont présentes, qu'il faudrait analyser, comme dans cet exemple :

Avec Laura nous avons interprété «Lundi rue Christine » avec de la dinette rose. Pour moi le théâtre a été la meilleure parti de cette sequence car on a pus pas seulement lire mais vivre le poeme s'était même se que j'ai preferé cette année a par la nouvelle [sic].

Dans « pas seulement lire mais vivre le poeme », une autre relation au texte est engagée : non une lecture orientant vers les « stratégies » traditionnelles de l'herméneutique, mais faisant advenir l'activité du poème, par la vie créative du lecteur qui le continue et fait émerger le sujet du poème.

De fait, les apports de cette démarche me semblent de trois ordres principaux :

- mobilisation des savoirs antérieurs, mise en relation et en perspective : genres, syntaxe, lexique ;

- stimulation de la motivation et de la curiosité, sécurisation ;

- stimulation d'une créativité transsubjective, avec appui sur les pairs, sous couvert d'un accueil bienveillant et ouvert de la part de l'enseignant, par une écoute constante de l'œuvre, dans son oralité globale.

Ce que résume pour une part cette conclusion extraite d'un carnet :

J'ai beaucoup apprécié l'ambiance en classe pendant la séquence, tout le monde a $\mathrm{pu}$ donner son oppinion, exprimer son avis. Mon regard sur la poésie a complétement changé, avant je pensais que la poésie était quelque chose de très réglementé, en proses ou vers et avec des rimes. Grâce à Apollinaire, j'ai compris que la poésie était quelque chose de très libre, on peut laisser notre imagination nous emporter dans la lecture et la création. L'auteur de Calligrammes voyait la beauté partout, et il réussissait à faire d'une chose banale une vraie œuvre. J'ai vraiment aimé pouvoir travailler sur des poèmes, les interpréter selon mon imagination. [sic]

\section{BIBLIOGRAPHIE}

APOLlinaire, G. (1918). «L'esprit nouveau et les Poètes ». En ligne : https://www.uni-due.de/ lyriktheorie/texte/1918_apollinaire.html.

APOLlinaiRe, G. (1966). Calligrammes. Poèmes de la paix et de la guerre (1913-1916). Paris : Gallimard. APOLLINAIRE, G. (2014). Calligrammes. Poèmes de la paix et de la guerre (1913-1916) : morceaux choisis. Paris : Larousse. 
BASSY, A.-M. et al. (1980). « Du calligramme ». Communication et langages 47 (1), p. 47-60. En ligne : https://www.persee.fr/doc/colan_0336-1500_1980_num_47_1_3460.

BORÉ, C. (2011). «L'énonciation des brouillons et la question du sujet scolaire ». Pratiques 149-150, p. 71-90. En ligne : https://journals.openedition.org/pratiques/1709.

BUTOR, M. (1966). « Préface ». In : Apollinaire, G., Calligrammes. Poèmes de la paix et de la guerre (1913-1916). Paris : Gallimard, p. 7-17.

CANVAT, K. (2007). « Lire du côté de chez soi. Réhabiliter la lecture "ordinaire" ». Études de Lettres 278, p. 19-52.

CHABANNE, J.-C. \& BUCHETON, D. (dirs) (2002). Parler et écrire pour penser, apprendre et se construire. L'écrit et l'oral réflexifs. Paris : Presses universitaires de France.

DEBON, C. (2004). «Calligrammes » de Guillaume Apollinaire. Paris : Gallimard.

DESSONS, G. (2004). L'art et la manière. Art, littérature, langage. Paris : H. Champion.

DESSONS, G. (2011). Le poème. Paris : A. Colin.

DESSONS, G. \& MESCHONNIC, H. (1998). Traité du rythme. Des vers et des proses. Paris : Dunod.

DUFAYS, J.-L. (2011). «Quel enseignement de la lecture et de la littérature à l'heure des

“compétences"? ». Pratiques 149-150, p. 227-248. En ligne : https://journals.openedition.org/ pratiques/1747.

FAVRIAUD, M. et al. (2009). « Nourrissage, amorçage et gestes professionnels dans la production poétique au cycle 2 de l'école primaire ». Repères 40, p. 175-199. En ligne : https:// journals.openedition.org/reperes/340.

faVriaud, M., Vinsonneau, M. \& poletto, M. (2017). Les chemins de poésie d'Alep. Poétique et didactique du dire-lire-écrire à l'école primaire. Limoges : Lambert-Lucas.

JORRO, A. \& CROCÉ-SPINELLI, H. (2010). « Le développement de gestes professionnels en classe de français. Le cas de situations de lecture interprétative ». Pratiques 145-146, p. 125-140. En ligne : https://journals.openedition.org/pratiques/1527.

LANGLADE, G. (2004). « Sortir du formalisme, accueillir les lecteurs réels ». Le français aujourd'hui 2 (145), p. 85-96. En ligne : https://www.cairn.info/revue-le-francais-aujourd-hui-2004-2page-85.htm.

LANGLADE, G. \& ROUXEL, A. (éds) (2004). Le sujet lecteur. Lecture subjective et enseignement de la littérature. Rennes : Presses universitaires de Rennes.

LAPLANTINE, C. (2011). Émile Benveniste, l'inconscient et le poème. Limoges : Lambert-Lucas.

MARTIN, S. (2013). « La voix, le sujet (subjectivation vs. subjectivisme). Emaz en "noyau d'énergie“». Voix et relation. En ligne : https://ver.hypotheses.org/602.

MARTIN, S. (2014). Poétique de la voix en littérature de jeunesse. Le racontage de la maternelle à l'université. Paris : L'Harmattan.

MARTIN, S. (2017). Voix et relation. Une poétique de l'art littéraire où tout se rattache. Taulignan : Delabre.

MARTIN, S. (2018). « Une poétique et une didactique des relations de Voix. Enjeux et perspectives pour l'enseignement et la recherche ». Carnets. Revue électronique d'études françaises de l'APEF 13. En ligne : https://journals.openedition.org/carnets/2651. 
MESCHONNIC, H. (1982a). Critique du rythme. Anthropologie historique du langage. Lagrasse : Verdier. MESCHONNIC, H. (1982b). « Qu'entendez-vous par oralité ? ». Langue française 56 (1), p. 6-23. En ligne : https://www.persee.fr/doc/lfr_0023-8368_1982_num_56_1_5145.

MESCHONNIC, H. (1995). Politique du rythme, politique du sujet. Lagrasse : Verdier.

MESCHONNIC, H. (2001). Célébration de la poésie. Lagrasse : Verdier.

MEschonnic, H. (2005). « Oui qu'appelle-t-on penser? ». In : Dessons, G., Martin, S. \& Michon, P. (dirs), Henri Meschonnic, la pensée et le poème. Paris : In Press, p. 251-266.

MESCHONNIC, H. (2006). Vivre poème. Liancourt : Dumerchez.

MINISTÈRE DE L'ÉDUCATION NATIONALE, DE L'ENSEIGNEMENT SUPÉRIEUR ET DE LA RECHERCHE (MÉNESR).

(2015). Programmes pour les cycles, p. 227-254. En ligne : http://cache.media.education.gouv.fr/ file/MEN_SPE_11/67/3/2015_programmes_cycles234_4_12_ok_508673.pdf. (consulté le 14/08/2018).

ROUXEL, A. (2007). « Pratiques de lecture : quelles voies pour favoriser l'expression du sujet lecteur?». Le français aujourd'hui 2 (157), p. 65-73. En ligne : https://www.cairn.info/revue-lefrancais-aujourd-hui-2007-2-page-65.htm.

VINSONNEAU, M. (2017). « Écrire de la poésie en classe de CP-CE1 en pensant la langue et l'apprentissage de la lecture ». In : Favriaud, M.,Vinsonneau, M. \& Poletto, M., Les chemins de poésie d'Alep. Poétique et didactique du dire-lire-écrire à l'école primaire. Limoges : Lambert-Lucas, p. 181-221.

\section{NOTES}

1. Pour un point sur le lien entre lecture littéraire et sujet lecteur, voir Dufays, 2011, p. 228-230.

2. Dans le carnet de recherche "voix et relation": https://ver.hypotheses.org/category/7theories-et-didactiques-de-la-litterature-une-question-de-voix/le-sujet (consulté le 10/08/2018).

3. Les élèves ont travaillé sur l'édition scolaire : G. Apollinaire (2014), édition présentée, annotée et commentée par P. Ouchy.

4. Quatre types d'activités sont proposées autour de la réénonciation: activités d'échelles (qui consistent à prélever des éléments dans les textes en les classant selon des critères à définir à chaque situation), activités d'oralisation, écriture créative, et recherche documentaire.

5. Voir M. Favriaud, M. Vinsonneau \& M. Poletto (2017, p. 133) pour un rappel des différents types de diction comme genre d'activité poétique centrale «constituant ce qu'on pourrait appeler une poétique de l'apprentissage de la littératie par la diction ». Cette affirmation pourrait recouper notre point de vue, prenant la mise en voix comme activité de réénonciation.

\section{RÉSUMÉS}

Partant du constat partagé que l'approche formaliste empêche la relation des élèves aux textes littéraires, l'article propose d'essayer de sortir de ce qui semble relever d'une aporie dans les 
théories du sujet lecteur qui maintiennent deux pôles séparés, texte et lecteur, quand le concept de sujet du poème, comme activité d'une transsubjectivation par les textes, permettrait d'envisager une troisième voie. Il présente les grandes lignes d'une séquence en classe de $4^{\mathrm{e}}$ menée avec Calligrammes. Poèmes de la paix et de la guerre (1913-1916) de G. Apollinaire, qui s'organise autour de la création d'un carnet de lecture, et cherche quels types d'indices et d'apports peuvent être envisagés, dans des activités où écrit et oral sont reliés, pour mieux porter attention à la signifiance comme force vive du langage. On observe, dans les réactions et les productions des élèves, les marques d'une expérience relationnelle à même de transformer les représentations de la poésie, de la langue, ainsi que les traces d'un devenir sujet.

Starting from the shared observation that the formalist approach prevents the relationship of pupils to literary texts, the article proposes to try to get out of what seems to be an aporia in the theories of the reader subject which maintain two separate poles, text and reader, when the concept of the poem subject, as an activity of a transsubjectivation through texts, would make it possible to envisage a third way. It presents the main lines of a sequence in the 4 th grade with Calligrammes. Poèmes de la paix et de la guerre (1913-1916) by G. Apollinaire, which is organised around the creation of a reading booklet, and looks for what types of clues and contributions can be envisaged, in activities where writing and speaking are linked, in order to pay better attention to the significance as the living force of language. We observe, in the reactions and the productions of the pupils, the marks of a relational experience able to transform the representations of poetry, language, as well as the traces of a becoming subject.

\section{INDEX}

Keywords : poem subject, transsubjectivation, oral, voice, reading booklet, Apollinaire (Guillaume)

Mots-clés : sujet du poème, transsubjectivation, oralité, mise en voix, carnet de lecture, Apollinaire (Guillaume)

\section{AUTEUR}

\section{FRÉDÉRIQUE COSNIER-LAFFAGE}

Université Sorbonne Nouvelle- Paris 3, Thalim, UMR 7172, F-75005, France/Université de Franche-Comté, Centre de linguistique appliquée, F-25000, France 